\title{
Case Report-Huge Lipoma in the Right Groin
}

\author{
Abdulla Mohammed Megally¹, M. A. Al-Katta1, Z. A. Mejalli' ${ }^{1}$ Hadi Mohammed Mujlli² \\ ${ }^{1}$ Department of General Surgery, Faculty of Medicine and Health Sciences, Sanaa University, Sanaa, Yemen \\ ${ }^{2}$ Thamar Medical College, Thamar University, Dhamar, Yemen \\ Email:dr.hadimujlli@gmail.com
}

How to cite this paper: Megally, A.M., Al-Katta, M.A., Mejalli, Z.A. and Mujlli, H.M. (2019) Case Report-Huge Lipoma in the Right Groin. Open Access Library Journal, 6: e5714.

https://doi.org/10.4236/oalib.1105714

Received: August 16, 2019

Accepted: September 27, 2019

Published: September 30, 2019

Copyright $\odot 2019$ by author(s) and Open Access Library Inc.

This work is licensed under the Creative Commons Attribution International License (CC BY 4.0).

http://creativecommons.org/licenses/by/4.0/ (c) (i) Open Access

\begin{abstract}
Lipoma is the most common soft tissue tumor, considered the universal tumor in the body. The differential diagnosis of a lipoma in the groin will include groin hernias, safina varex, lymphadenopathy, hydrocele and sarcoma. Due to the similarity of clinical symptoms and signs, the differentiation between inguinal, femoral hernias and groin lipoma is difficult. To avoid the high risk of intraoperative complications, correct diagnosis is an indication. We are reporting a case with huge lipoma arisen from the right groin mainly from right labium major of a 19-year-old single girl grown in a relatively short period to reach dimensions of a football and weighing 7200 grams. Painless, pedunculated tumor arose from labium major to the level of the knee, asymptomatic except unsightly huge mass, which treated successfully by surgical excision without complications and proved benign with no malignant changes detected.
\end{abstract}

\section{Subject Areas}

Medical Genetics, Nursing, Public Health, Surgery \& Surgical Specialties

\section{Keywords}

Groin Lipoma, Huge Lipoma of Labium Majora, Soft Tissue Tumour

\section{Introduction}

Lipomatous tumor is the most common tumor of the soft tissue tumors, and can arise in any organ of the body where fat cells normally exist, therefore named, the universal tumor [1] [2] [3]. Commonly, lipomas may arise subcutaneously, subfascial, intermuscular, subperiostial, subsynoveal, intra-articular, intraglandular, subserosal or submucosal locations may exist. The frequent locations of lipoma are the proximal extremities, trunk and groin [4] [5]. Clinically, lipoma presents as painless subcutaneous swellings of variable sizes commonly of long 
history duration. The diagnosis depends on careful history and examination, but ultrasound, computed tomography (CT) scanning or magnetic resonance image (MRI) could diagnose and differentiate lipoma from other soft tissue swellings, hernias or cysts. CT provides useful information on the origin of the tumor. Histopathology is essential to differentiate benign from malignant liposarcoma. The main treatment of lipoma is completely surgical excision. Completely surgical excision prevents local recurrence. In this presentation, we report a case with huge lipoma of the right groin progressed in short time with its diagnostic and treatment aspects.

\section{Case Report}

A nineteen years old single female patient came to our surgical outpatient department of Al-Thawrah Modern General Hospital, a teaching hospital, in Sanaa, Yemen, with a seven months history of huge painless swelling pedunculated from the right groin reaching to the level of the knee joints. The swelling rapidly progressed in size to reach a football-size within seven months duration. The patient appeared healthy, no other swelling detected. The patient denied any difficulty on walking, in spite of the huge, heavy, pedunculated, freely mobile swelling. There was no past history of trauma, foreign body impaction, fever or change in color of the skin over the swelling. The tumor mass noticed to double in size per month without any disability or illness. On examination, there was tumor of $37 \times 28 \times 25 \mathrm{~cm}$ dimensions hanging from the right labium major dawn to the level of knee joint. The tumor had well defined edges, soft to firm in consistency not tender, no impulse with coughing and we can get above the mass on palpation, excluding a hernia (Figure 1).

The swelling was not attached to overlying skin or underlying structures with visible superficial dilated veins. There was no lymphadenopathy in the groin. The cardiovascular system and chest were clear. Laboratory investigations were within normal values, with hemoglobin $14 \mathrm{~d}$ normal renal and liver function tests. There were no hormonal abnormalities detected. Average body built and
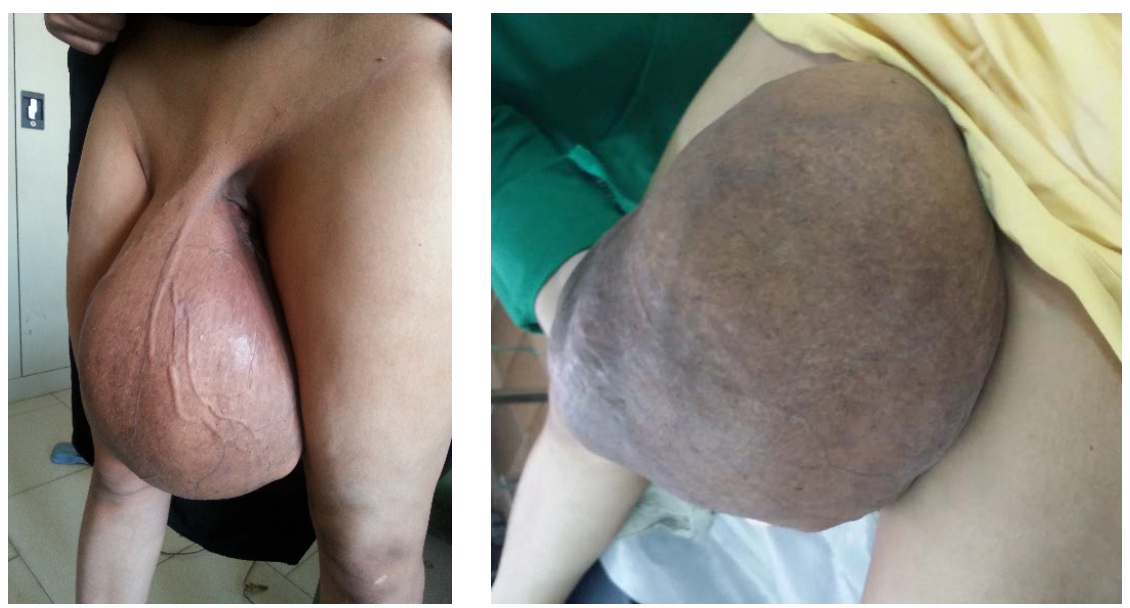

Figure 1. Giant lipoma of right groin in erect and supine. 
texture with well-developed breasts. Abdominal and pelvic ultrasonography and chest x-ray revealed no abnormalities. Pelvic CT and MRI were done, and we show well-defined and capsulated mass lesion (Figure 2 \& Figure 3).

Excision biopsy is taken to verify types of this soft tissue tumor, which proved a benign lipoma and excluded sarcomatous changes. Surgical excision performed under general anesthesia and the specimen transected to see the component of the mass, which proved a pure, well capsulated lipoma (Figure 2 \& Figure 3), meticulous hemostasis was secured and the wound closed without any skin tension, after placing suction drain left for 48 hours. Post-operative recovery went smoothly without complications and patient discharged on the seventh post-operative day. The surgical specimen was $38 \times 35 \times 28 \mathrm{~cm}$, well capsulated fatty mass with its covering skin; transection revealed some heterogeneity of necrotic inflammatory tissue of bad smell, anaerobic infection in the previous

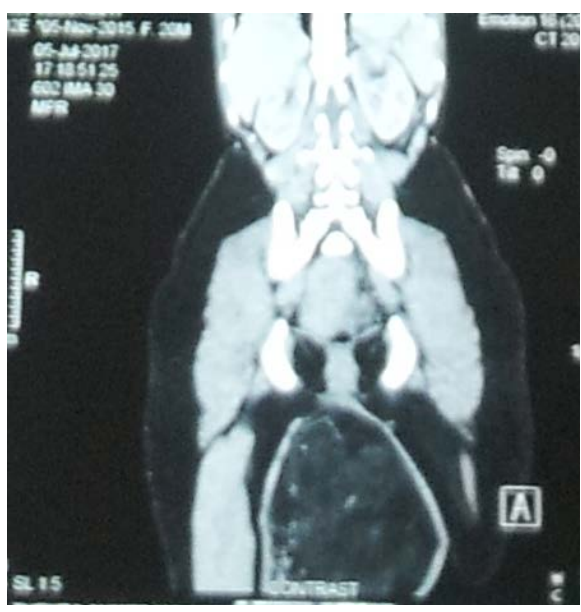

Figure 2. CT scan show pedunculated mass lesion.

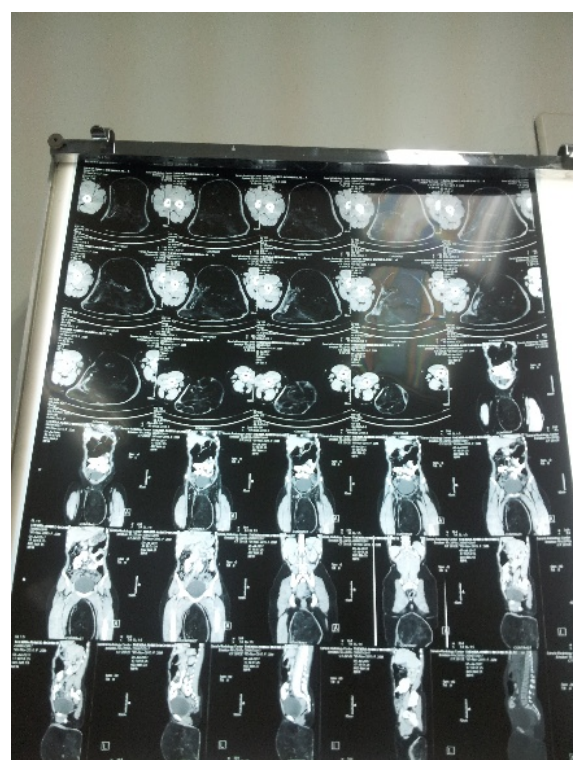

(a)

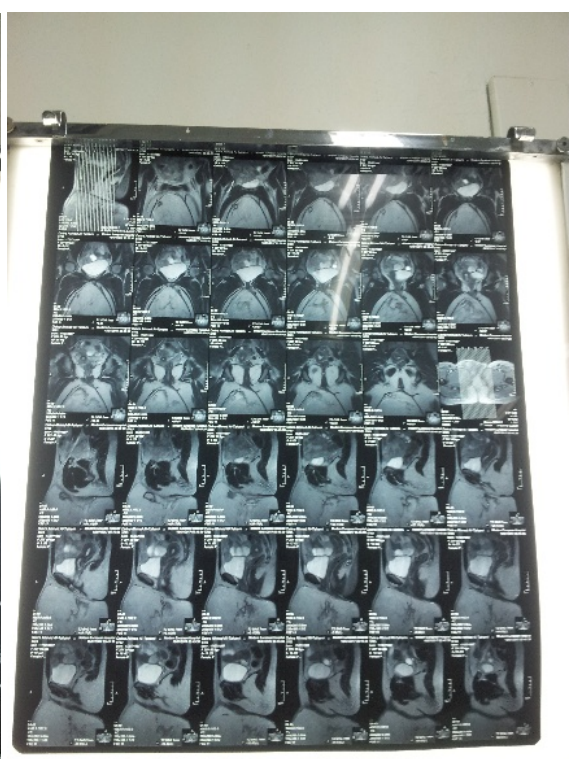

(b)

Figure 3. (a) CT of tumour, (b) MRI of tumour. 
excisional biopsy and sent again for histopathology and histochemistry aiming to find any accelerating growth factors or sarcomatous changes (Figure 4).

\section{Discussion}

Primary benign tumors of the groin may arise from connective tissue, muscle, fat, blood vessels and lymphoid tissue [2] [3]. Lipomata is the most common soft tissue tumors, which can arise in any organ of the body of variable sizes [1]. The majority of soft tissue tumors are benign and arise on the extremities, the lower limb, pelvic girdle, and trunk being the most frequent sites. Lipomas vary in size from small, giant to huge swellings. For lipoma to be giant, it should be $10 \mathrm{~cm}$ or more in diameter and a minimum weight 1000 gram, therefore, our case considered super giant as $37 \mathrm{~cm}$ diameter and $7.2 \mathrm{~kg}$ weight (huge). The majority of patients present complaining of a swelling that has been gradually enlarging over several years in case of lipoma, or several months only in case of liposarcoma, but our case was an exception of huge lipoma developed during seven months only. Pain and disability are often absent, so there may be a substantial delay before presentation, the tumor being remarkably large when first examination. Symptoms such as dragging sensation, functional limitations, weakness of legs or compression on neighboring structures (arteries, veins, muscles or lymphatic) may attribute to other causes such as prolapsed intervertebral disc. The clinical examination and investigations may diagnose a fatty tissue swelling; however, a swelling in the groin can be mistaken as groin hernia (inguinal or femoral), hydrocele or lymphoma. Some tumors display features strongly suggestive of malignancy but more often the distinction between a benign and malignant lesion is difficult to make on simple clinical grounds. Imaging techniques may diagnose tumors from hernias but not with a $100 \%$ specificity or sensitivity. Computed tomographic (CT) scanning and magnetic resonance image (MRI) were performed to help in arriving at diagnosis and features that suggest nature of the swelling, which described a well capsulated, circumscribed, heterogeneous fatty mass. It is essential to obtain histological confirmation of
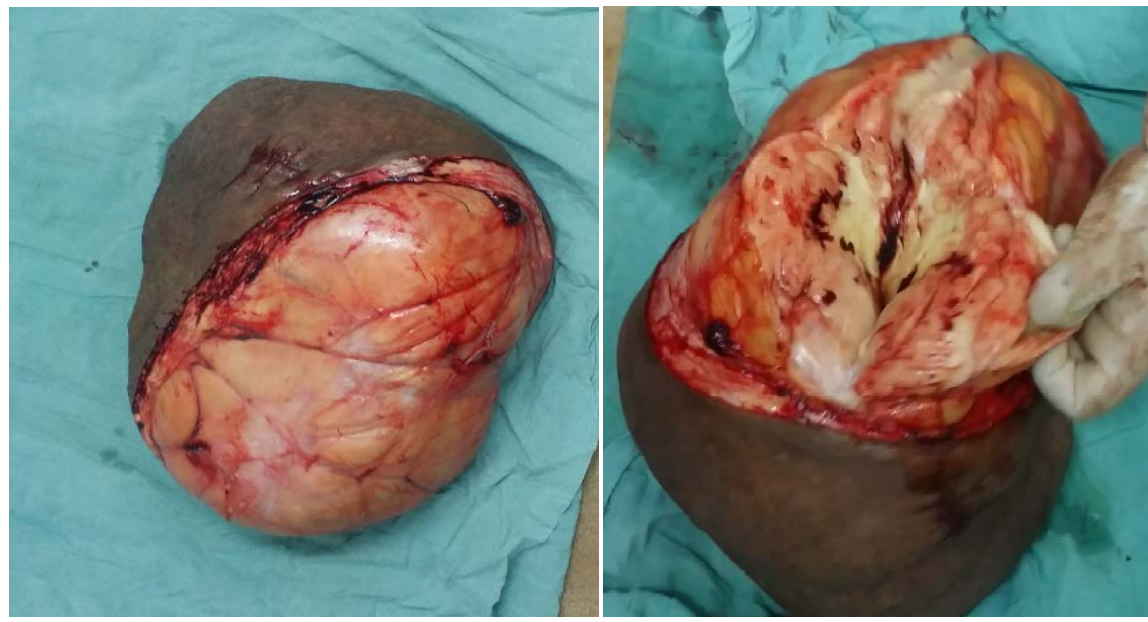

Figure 4. Excised tumour $(35 \times 30 \times 28 \mathrm{~cm})$. 
the diagnosis before planning treatment. It is possible to make the diagnosis from a fine needle aspiration cytology (FNAC) or true-cut tissue biopsy, which can make accurate diagnosis in over $90 \%$ of cases, but this, is wholly unreliable. This is because liposarcoma may contain areas of normal fatty tissue as a result of their lake of homogeneity. With modern histochemical techniques, the subtype and grade of the tumor can be correctly specified in more than $80 \%$ of samples. It was postulated that any soft tissue tumor its size greater than $5 \mathrm{~cm}$ should be considered a malignant until proved otherwise, however, in our case the size was greater than $5 \mathrm{~cm}$, suggest malignant, but the histopathology confirmed benignity of lipoma despite huge size grown in short time. Classically, the main treatment of giant lipoma is wide surgical excision, because these tumors may transform to malignant liposarcoma. Successful wide excision was done in our case. In spite of amputation of the tumor with its covering skin, there is a risk of local recurrence after a variable time period, therefore, a long-term follow-up is recommended in such patients.

\section{Conclusion}

According to our finding, the size of the lipoma presented in our case has little been described before in national medical literature, but there are international case reports which are published. Whereas MRI and CT have confirmed the diagnosis of fatty tissue, the final confirmation is made by histopathological. Surgical excision is the treatment of choice to get rid of symptoms and to exclude any malignancy changes and provides definite tissue diagnosis.

\section{Conflicts of Interest}

The authors declare no conflicts of interest regarding the publication of this paper.

\section{References}

[1] Mentzel, T. and Fletcher, C.D. (1995) Lipomatous Tumors of Soft Tissue: An Update. Virchows Archiv, 427, 353-363. https://doi.org/10.1007/BF00199383

[2] Browse, N. (1990) Hernia. In: Browse, N., Ed., An Introduction to the Symptoms and Sings of Surgical Disease, Edward Arnold, London, 272-273.

[3] Wang, T. and Vaipeyl, R. (2015) Hernia Sacs: Is a Histological Examination Necessary? Journal of Clinical Pathology, 66, 104-106.

[4] Castea, R., Vasiliu, E., Zarnscu, N.O., Hasouna, M. and Neagu, S. (2011) Large Thigh Liposarcoma-Diagnosis and Therapeutic Features. Journal of Medicine and Life, 4, 184-188.

[5] Akinkunmi, M., Balogun, B., Awosanya, G., Fadeyibi, I., Benebo, A.S., Soyem, S. and Obafunwa, J. (2009) Giant Fibrolipoma of the Thigh in a Nigerian Woman: A Case Report. The Internet Journal of Radiology, 12. 